\title{
Injection Laryngoplasty: Techniques and Choices of Fillers
}

\author{
Jonathan B. Salinas • Dinesh K. Chhetri
}

Published online: 23 January 2014

(c) Springer Science+Business Media New York 2014

\begin{abstract}
Since it was first introduced in 1911, injection laryngoplasty has evolved into a versatile technique for the treatment of dysphonia in patients with a glottic insufficiency. Currently, there are a number of materials available for injection augmentation with duration of efficacy that may last from weeks to years. Furthermore, the various approaches available for injection have provided for injection laryngoplasty to be performed accurately in both the office and operating room.
\end{abstract}

Keywords Injection laryngoplasty - Glottic insufficiency · Dysphonia · Hoarseness · Vocal fold augmentation · Vocal fold paresis $\cdot$ Vocal fold paralysis $\cdot$ Presbylaryngis

\section{Introduction}

Injection laryngoplasty is a technique aimed at augmenting and medializing the vocal fold in order to decrease the glottic insufficiency caused by a variety of conditions such as vocal cord paralysis, paresis, or atrophy. Injection laryngoplasty was first introduced in 1911 by Bruening as a transoral procedure performed under general anesthesia [1]. It represents the oldest surgical technique developed for the management of vocal fold insufficiency [2••]. Over time, the technique has developed primarily into an office-

J. B. Salinas $(\varangle) \cdot$ D. K. Chhetri

Voice, Airway, and Swallowing Disorders, Department of Head and Neck Surgery, David Geffen School of Medicine, University of California, Los Angeles, CA, USA

e-mail: jsalinas@mednet.ucla.edu

D. K. Chhetri

e-mail: dchhetri@mednet.ucla.edu based procedure. In 1985, Ward et al. [3] introduced the office-based transcutaneous approach, which allowed for injection of the vocal fold under local anesthesia while using video laryngoscopy for visualization. This approach provided vocal fold augmentation in patients with difficult anatomy and trismus, in whom a transoral approach was difficult to perform. The initial description of this technique mentioned a trans-cricothyroid membrane approach; however, transthyroid cartilage and trans-thyrohyoid membrane approaches have also been used $[4 \bullet, 5]$. Currently, office-based approaches are most commonly used for vocal cord augmentation $[6,7 \bullet]$.

A variety of materials are currently available for injection laryngoplasty, each allowing a wide range of duration of the medialization effect. For instance, while medialization performed with carboxymethycellulose (Radiesse Voice Gel $^{\circledR}$, Merz Aesthetics, Inc., Franksville, WI, USA) may last for 4-12 weeks, the results after injecting autologous fat may last for years [8].

This article describes the indications, patient selection criteria, techniques, and materials used in injection laryngoplasty.

\section{Indications and Patient Selection Criteria}

Injection laryngoplasty may be performed for a number of conditions that result in a glottic insufficiency and perceptual changes in the quality of the voice. These conditions include vocal cord paralysis, paresis, atrophy, bowing, and scarring. It is important to note that there are many medical conditions that may result in the development of a glottic gap, including thyroid cancer, laryngeal cancer, Parkinson's disease, rheumatoid arthritis, etc. For this reason, it is crucial for the otolaryngologist to perform 
a thorough evaluation to diagnose and treat the primary etiology of the glottic insufficiency before initiating treatment with injection laryngoplasty.

\section{Awake Injection Laryngoplasty}

The clinician should be aware that awake injection laryngoplasty has an initial learning curve that ultimately leads to excellent results with experience. Awake injection laryngoplasty should be the primary technique initially considered for vocal fold medialization in all patients. Nevertheless, there are situations in which this technique has an increased advantage for a successful outcome. For instance, there is an advantage in performing an awake injection laryngoplasty in patients with significant comorbidities that place them at risk for complications associated with general anesthesia [9]. In the same way, if the patient has symptoms that are not severe enough to merit the inconvenience and risk of general anesthesia, an awake injection is favored [9]. Furthermore, an awake procedure should be considered when there is an uncertain benefit to the injection or when the diagnosis itself is still in question. In these cases, a trial augmentation may allow the patient to feel the degree of voice improvement to make an informed decision regarding permanent medialization [9].

The avoidance of the risk of general anesthesia is especially relevant in patients that need augmentation secondary to atrophy, since these patients tend to be elderly. This is especially important since injection laryngoplasty and surgical medialization laryngoplasty may yield similar voice outcomes after a 6-month follow-up period [10].

Awake injection laryngoplasty also offers the advantage of allowing for the immediate assessment of the injection result, based on the voice quality, airway patency, location, and diffusion of the injected material. Sulica et al. [9] have point out that continuous visualization of the dynamic larynx allows for optimal injection placement and quantity to reach an adequate functional outcome and avoid complications. Furthermore, the use of videostroboscopy before and after injection allows for the immediate assessment of the glottic closure.

There is also a cost and time advantage associated with an awake injection laryngoplasty that should be considered. For instance, the time required to perform the procedure including consent and placement of topical anesthesia is usually below $30 \mathrm{~min}$, eliminating the time required for anesthesia preparation, induction, and operating room setup. Of course, there is also a significant cost effectiveness advantage in eliminating the hospital and anesthesia costs. In fact, Bove et al. [11•] used a predictive model to describe a net savings accrued to the healthcare economy of more than $\$ 40$ million per year, if there was a broad acceptance and application of office-based injection laryngoplasty.

Injection Laryngoplasty Under General Anesthesia

This technique continues to play an important role as it allows for better technical control of needle location and injectable distribution as compared to an injection in an awake patient. Furthermore, having the patient under general anesthesia allows for additional surgical procedures, such as autologous fat or fascia harvest. In cases of vocal cord scarring, injection in the operating room permits for microsurgical dissection to further evaluate this diagnosis [9]. Finally, anxious or uncooperative patients are best treated under general anesthesia.

\section{Materials}

The ideal material to be used for injection should be biocompatible and inert to decrease the risk of local tissue reaction or fibrosis [8]. Also, the material should be easy to use and have a low cost. Furthermore, it should be durable and resistant to resorption or migration, while it maintains the normal viscoelasticity of the vocal cord after injection [8].

The materials currently available for injection laryngoplasty include hyaluronic acid-based materials (Restylane ${ }^{\circledR}$, Medicis Aesthetics, Scottsdale, AZ, USA, and Juvederm $^{\circledR}$, Allergan, Irvine, CA, USA), autologous fat, carboxymethycellulose (Radiesse Voice Gel ${ }^{\circledR}$, Merz Aesthetics, Inc., Franksville, WI, USA), micronized acellular dermal matrix $\left(\right.$ Cymetra $^{\circledR}{ }^{\circledR}$, Life Cell Corp., Branchburg, NJ, USA), and calcium hydroxylapatite (Radiesse Voice, Merz Aesthetics, Inc., Franksville, WI, USA) [2••]. Older materials, such as Teflon ${ }^{\circledR}$ (DuPont, Wilmington, DE, USA), are no longer used because of complications associated with them, such as granuloma formation. Many of these products were developed as dermal fillers and are not specifically approved for use in the larynx; however, there is wide acceptance of off-label use of these products among otolaryngologists.

Since there is no perfect material on the market, it is important to understand the benefits and disadvantages of the materials available to select the optimal material for the situation at hand.

Carboxymethycellulose (Radiesse Voice Gel ${ }^{\circledR}$, Merz Aesthetics, Inc., Franksville, WI, USA)

Carboxymethycellulose is prepared as a gel, composed of sodium carboxymethylcellulose, glycerin, and water. This 
material has the advantage of being inert, and therefore no allergic testing is needed prior to its use [12]. Also, the material is ready to use without the need for preparation or harvesting. This material can last 1-3 months [12].

\section{Calcium Hydroxylapatite (Radiesse Voice ${ }^{\circledR}$, Merz Aesthetics, Inc., Franksville, Winconsin, USA)}

This material is a mineral found in human bone and teeth. The injectable form is a combination of calcium hydroxylapatite microspheres suspended in carboxymethylcellulose carrier gel. Kwon et al. [13] and Rosen et al. [14] have shown that this material can last up to 12 months, while Carroll et al. [15] showed an average duration of 18.6 months after injection.

\section{Collagen-Derived Products}

A number of collagen-derived products are available for injection. Historically, these materials were engineered from either bovine or human collagen. Zyplast ${ }^{\circledR}$ (Inamed Aesthetics, Fremont, CA, USA) and Zyderm ${ }^{\circledR}$ (McGhan Medical Corp., Fremont, CA, USA) were derived from bovine collagen and lasted for up to 4 months [8]. Cosmoplast ${ }^{\circledR}$ (Allergan, Irvine, CA, USA) and Cosmoderm ${ }^{\circledR}$ (Allergan, Irvine, CA, USA) were derived from human collagen. These materials are no longer available for clinical use.

Cymetra ${ }^{\circledR}$ (Life Cell Corp., Branchburg, NJ, USA) is not specifically a collagen product but is similar in nature. It is derived from processed human cadaveric dermal tissue; therefore, it has the potential risk of pathogen transmission from the donor tissue, although so far no infection transmissions have been reported [8]. Cymetra ${ }^{\circledR}$ has a variable average duration from 2 to 3 months to more than 1 year $[8,16]$.

\section{Hyaluronic Acid}

This material is a natural polysachharide that forms part of the extracellular matrix. This polysachharide is also found in the lamina propia of the vocal cords and helps in the repair of its tissue, providing a synthetic building block for the extracellular matrix [8]. The hyaluronic acid version available for injection is cross-linked, making it more durable. Rheologic studies have shown that hyauronic acidbased materials have a viscoelastic profile that most closely resembles that of the human vocal fold [17]. Restylane and Hylan b Gel are commercially available preparations used off-label in the larynx that are made of cross-linked chains of hyaluronic acid. These materials may last approximately 4-6 months [8].
Autologous Fat Injection

Autologous fat has been shown to give a longer lasting medialization effect after injection as compared to other materials. Although its duration has been reported to be over 26 months, it actual duration is variable because of its unpredictable rate of resorption [8]. The fat tissue is usually harvested from the subcutaneous tissues of the abdominal wall. Because of its autologous nature, it is biocompatible and safe for use [8]. Also, it is a material that is close to the viscoelasticity of the vocal fold cover layer [18]. One of the major disadvantages of fat injection is the prolonged harvest time. Furthermore, the unpredictability of fat survival causes variability in the outcomes of fat injections. Although there have been reports of donor site hematomas, poor voice quality due to over-injection, and fat extrusion, the complication rate is low. Another disadvantage of this procedure is the fact that the patient needs to undergo general anesthesia. In addition, patients tend to suffer a prolonged postoperative dysphonia for up to a few weeks because of the needed over-injection.

\section{Technique}

There are three major approaches to injection laryngoplasty: percutaneous, transoral, and microlaryngoscopy.

\section{Percutaneous Approach}

Injection laryngoplasty through a percutaneous approach can be performed following a trans-cricothyroid membrane, trans-thyroid cartilage, or trans-thyrohyoid route $[2 \bullet \bullet, 4 \bullet]$. The initial step in preparing for a percutaneous injection is localizing the laryngeal landmarks by palpation. The most important landmarks are the thyroid notch, inferior border of thyroid cartilage, cricoid cartilage, and cricothyroid membrane. If desired, $0.5-1.0 \mathrm{~mL}$ of local anesthetic can be infiltrated with a $1-\mathrm{mL}$ syringe and 30-gauge needle over the cricothyroid membrane for the trans-cricothyroid approach, over the lower border of the thyroid lamina for the transthyroid approach, and over the thyroid notch for the trans-thyrohyoid approach. The injection is carried out under direct visualization with the help of a flexible nasolaryngoscope connected to a video monitor.

\section{Transcricothyroid Approach [2••]}

Injection laryngoplasty through the cricothyroid membrane is the most common approach used [8]. In this approach, the cricoid and cricothyroid membrane are first palpated. Next, the index finger of the non-injecting hand is placed 
over the cricothyroid membrane at the level of the inferior thyroid ala. The 27-gauge injection needle is inserted at the inferior border of the thyroid cartilage, approximately $5 \mathrm{~mm}$ lateral to the midline in a perpendicular direction to the thyroid ala. This is advanced until the needle tip makes contact with the cartilage. At this point, the needle tip is pushed inferiorly to guide the tip under the inferior border of the thyroid cartilage. It is important to use the tactile feedback of the needle traveling underneath the thyroid border, as this will confirm that the needle will then be guided into the paraglottic space. With the needle tip in this position, making sure not to retract the needle, the injecting hand can then be used to create a bend in the needle to an angle of $30^{\circ}$ at the hub. This is done by pushing the syringe against the neck skin while the needle tip is pointed superiorly and laterally. At this point, the needle is slowly advanced 5-10 $\mathrm{mm}$ in this position, aiming superiorly and laterally. While watching on the video monitor, the needle is manipulated gently while injecting a small amount of the augmenting material to help identify the intralaryngeal position of the tip. Next, the material is injected while visualizating for adequate medialization of the vocal fold.

\section{Transthyroid Cartilage Approach [2••]}

For this approach, the laryngeal landmarks are also palpated, and the index finger is placed at the level of the cricothyroid membrane. The needle is placed perpendicular to the thyroid cartilage, approximately $5 \mathrm{~mm}$ lateral to the midline and $2-3 \mathrm{~mm}$ above the inferior border. As the vocal fold level usually lies halfway between the thyroid notch and the inferior border of the thyroid cartilage, if the landmarks are palpable, one can make a mental picture of the approximate target for injection. The needle can then be placed at a vertical level at a midpoint between the superior vocal fold and the inferior thyroid cartilage border.

Gentle pressure is applied until the needle goes through the cartilage. At this point, the needle is bent slightly at the hub to point laterally toward the paraglottic space. The injection can then be performed while observing for medialization on the video monitor.

\section{Transhyoid Approach [3]}

After infiltrating local anesthetic over the thyroid notch and the thyrohyoid membrane area, the syringe is filled with $4 \%$ lidocaine in order to inject through thyrohyoid membrane into the airway. Following this, under direct visualization with a nasolaryngoscope, the syringe filled with the injectable material is attached to the appropriate needle. The needle is inserted immediately above the thyrohyoid notch and through the subcutaneous tissues at a downward angle, passing though the pre-epiglottic space and entering the larynx at the level of the petiole of the epiglottis. Next, the needle is slowly advanced to the preferred injection site in a unilateral or bilateral fashion.

\section{Transoral Injection [8]}

This approach can be performed in the operating room or office setting under direct visualization with the help of a nasolaryngoscope. First, the patient's tongue is held with gauze with one hand while a syringe with the injectable material is held on the other hand. Usually a $25-\mathrm{cm}-1$ long needle with a 16-gauge malleable shaft and a 25-gauge needle tip is used for injection. The needle is bent appropriately to be able to be directed through the pharynx and into the larynx. While an assistant holds the flexible nasolaryngoscope, the needle is inserted orally and aimed toward the appropriate vocal fold. The material should be injected lateral to the vocal cord at the level of the vocal process.

\section{Transnasal, Endoscopic [19]}

This approach can be performed in the office setting under topical anesthesia. A flexible telescope with a working channel is introduced transnasally to the level of the laryngopharynx. Anesthesia of the larynx is obtained by topical application of $4 \%$ lidocaine solution. A sclerotherapy needle is loaded with the injection material and then passed through the working channel of the telescope and directed permucosally into the paraglottic space lateral to the affected vocal fold for injection augmentation. This technique is limited by the rather long length of the injection tubing that increases the resistance to the flow of material while injecting, thus requiring a fair amount of injection pressure to push out the material. Materials of higher viscosity cannot be injected with this technique, but it has been used successfully with many of the materials used in the office setting.

\section{Microlaryngoscopy Approach [8]}

This approach is done under general anesthesia and in a similar fashion as the transoral approach. After the patient is intubated, the head of the bed is turned $90^{\circ}$ from the anesthesiologist. Next, the larynx is exposed and suspended with the use of rigid laryngoscopy. Using an operating microscope to visualize the vocal cords, a $25-\mathrm{cm}$-long needle with a 25-gauge malleable tip was directed through the rigid laryngoscope. As in the transoral approach, the needle is aimed laterally to the vocal fold at the level of vocal process. 


\section{Complications}

The complication rates seen in both awake and asleep injections are very low. These complications usually are related to respiratory difficulty, misplacement of the material, or a reaction to the material. In terms of the potential misplacement of the material, this particularly occurs in the form of superficial injection into the subepithelial lamina propria space [9]. This can represent a problem, since currently there are no materials with appropriate rheologic properties for injection in the subepithelial plane. Injection into this plane is likely of little consequence when short-lived materials are used. However, in the longer lasting materials, subepithelial injections can have significant consequences for the vocal fold vibration secondary to the inflammatory reaction the material may produce [9]. For instance, subepithelial injection of a material such as calcium hydroxylapatite will result in inflammation and stiffness, which may be permanent [20]. It is interesting to note that females are at a higher risk of having a superficial injection secondary to their smaller larynx [21]. This places an emphasis on injection accuracy. It is important to know the exact position of the needle at all times prior to starting injection. Furthermore, if the injection is technically difficult, one can choose to use a short-lasting injectable to minimize the risk of permanent hoarseness from accidental misplacement. Finally, although injection laryngoplasty is accepted as a generally safe technique, the clinician should always be aware of the possibility of airway obstruction, airway hemorrhage, aspiration, or injury to the adjacent laryngeal structures.

\section{Conclusion}

Injection laryngoplasty has evolved into a versatile technique that offers the surgeon the ability to treat patients with dysphonia secondary to glottic insufficiency. Recently, in-office injection laryngoplasty has gathered significant popularity since it can be performed without the need for general anesthesia, making it a preferred approach for patients with medical comorbidities. Furthermore, inoffice injections have been shown to be cost effective, since they avoid the costs associated with general anesthesia and the operating room. However, it is important to note that injection laryngoplasty under general anesthesia continues to be an important approach in patients with anxiety or who are otherwise uncooperative.

It is crucial for the clinician to be familiar with the different approaches available for injection laryngoplasty in order to optimize the accuracy of the injection. In the same way, the clinician needs to be well versed in the different materials available for injection in order to select the ideal material for an optimal outcome based on the needs of the patient.

\section{Compliance with Ethics Guidelines}

Conflict of Interest Jonathan B. Salinas and Dinesh K. Chhetri declare no conflicts of interest.

Human and Animal Rights and Informed Consent This article does not contain any studies with human or animal subjects performed by the authors.

\section{References}

Papers of particular interest, published recently, have been highlighted as:

- Of importance

•- Of major importance

1. Brunings W. Uber eine neue Behandlungsmethode der Rekurrenslahmung. Verhandl Ver Dtsch Laryngol. 1911;17:93.

2. • Chhetri DK, Jamal N. Percutaneous injection laryngoplasty. Laryngoscope. 2013. doi:10.1002/lary.24417. This article details the trans-cricothyroid membrane and trans-thyroid cartilage approaches for injection laryngoplasty.

3. Ward PH, Hanson DG, Abemayor E. Transcutaneous teflon injection of the paralyzed vocal fold: a new technique. Laryngoscope. 1985;95:644-9.

4. - Amin MR. Thyrohyoid approach for vocal fold augmentation. Ann Otol Rhinol Laryngol. 2006;115:699-702. This article details the thyrohyoid approach for injection larynoplasty.

5. Lee SW, Kim JW, Koh YW, Shim SS, Son YI. Comparative analysis of efficiency of injection laryngoplasty technique for with or without neck treatment patients: a transcartilaginous approach versus the cricothyroid approach. Clin Exp Otorhinolaryngol. 2010;3:37-41.

6. Luu Q, Tsai V, Mangunta V, Berke GS, Chhetri DK. Safety of percutaneous injection of bovine dermal crosslinked collagen for glottis insufficiency. Otolaryngol Head Neck Surg. 2007;136(3): 445-9.

7. - Mallur PS, Rosen CA. Office-based laryngeal injections. Otolaryngol Clin North Am. 2013;46:85-100. This article details the setup and technique used for office-based laryngeal injections.

8. Phua CQ, Mahalingappa Y, Homer J, Karagama Y. Injection laryngoplasty. The Otorhinolaryngologist. 2013;6(2):111-8.

9. Sulica L, Rosen CA, Postma GN, Simpson B, Amin M, Courey $\mathrm{M}$, Merati A. Current practice in injection augmentation of the vocal folds: indications, treatment principles, techniques, and complications. Laryngoscope. 2010;120(2):319-25.

10. Vinson KN, Zraick RI, Ragland FJ. Injection versus medialization laryngoplasty for the treatment of unilateral vocal fold paralysis. Laryngoscope. 2010;120:1802-7.

11. - Bove MJ, Jabbour N, Krishna P, et al. Operating room versus office-based injection laryngoplasty: a comparative analysis of reimbursement. Laryngoscope. 2007;117:226-300. This article compares the reimbursement of injection laryngoplasty done in the operating room versus the office. It also describes a national predictive model with potential savings to the insurance industry of over $\$ 40$ million per year if office-based injection laryngoplasty is widely used. 
12. Kwon TK, Rosen CA, Gartner-Schmidt J. Preliminary results of a new temporary vocal fold injection material. J Voice. 2005;19(4): $668-73$.

13. Kwon TK, An SY, Ahn JC, Kim KH, Sung MW. Calcium hydroxylapatite injection laryngoplasty for the treatment of presbylaryngis: long-term results. Laryngoscope. 2010;120(2): 326-9.

14. Rosen CA, Gartner-Schmidt J, Casiano R, Anderson TD, Johnson $\mathrm{F}$, Remacle $\mathrm{M}$, et al. Vocal fold augmentation with calcium hydroxylapatite: twelve-month report. Laryngoscope. 2009; 119(5):1033-41.

15. Carroll TL, Rosen CA. Long-term results of calcium hydroxylapatite for vocal fold augmentation. Laryngoscope. 2011;121(2): 313-9.

16. Tan M, Woo P. Injection laryngoplasty with micronized dermis: a 10 -year experience with 381 injections in 344 patients. Laryngoscope. 2010;120(12):2460-6.
17. Caton T, Thibeault SL, Klemuk S, Smith ME. Viscoelasticity of hyaluronan and nonhyaluronan based vocal fold injectables: implications for mucosal versus muscle use. Laryngoscope. 2007; 117(3):516-21.

18. Chan RW, Titze IR. Viscosities of implantable biomaterials in vocal fold augmentation surgery. Laryngoscope. 1998;108(5): 725-31.

19. Trask DK, Shellenberger DL, Hoffman HT. Transnasal, endoscopic vocal fold augmentation. Laryngoscope. 2005;115: 2262-5.

20. Chheda NN, Rosen CA, Belafsky PC, Simpson CB, Postma GN. Revision laryngeal surgery for the suboptimal injection of calcium hydroxylapatite. Laryngoscope. 2008;118:2260-3.

21. Jamal N, Mundi J, Chhetri DK. Higher risk of superficial injection into the lamina propria layer during injection laryngoplasty in women. Am J Otolaryngol. 2013 (accepted). 